\title{
LOCAL FOURIER TRANSFORMS AND RIGIDITY FOR D-MODULES*
}

\author{
SPENCER BLOCH ${ }^{\dagger}$ AND HÉLÈNE ESNAULT ${ }^{\ddagger}$
}

To Armand Borel, in memoriam

\begin{abstract}
Local Fourier transforms, analogous to the $\ell$-adic local Fourier transforms [14], are constructed for connections over $k((t))$. Following a program of Katz [12], a meromorphic connection on a curve is shown to be rigid, i.e. determined by local data at the singularities, if and only if a certain infinitesimal rigidity condition is satisfied. As in [12], the argument uses local Fourier transforms to prove an invariance result for the rigidity index under global Fourier transform. A key technical tool is the notion of good lattice pairs for a connection [5].
\end{abstract}

1. Introduction. In an important article, G. Laumon [14] applied the $\ell$ adic Fourier transform to study epsilon factors associated to $\ell$-adic sheaves on curves over finite fields. As a key tool, he defined local Fourier transforms $\mathcal{F}(0, \infty), \mathcal{F}(\infty, 0), \mathcal{F}(\infty, \infty)$ for $\ell$-adic sheaves on $\operatorname{Spec}_{\mathbb{q}}((t))$. Recently, we applied his ideas to study epsilon factors associated to holonomic $\mathcal{D}$-modules on curves. The purpose of this paper is to develop local Fourier transforms for meromorphic connections over Laurent series fields. We show that these have properties precisely analogous to the Laumon local $\ell$-adic local Fourier transforms.

As an application of our construction, we consider the index of rigidity of meromorphic connections as defined by N. Katz [12]. A local system on $\mathbb{P}^{1} \backslash S$ over the complex numbers, where $S$ is a finite set of points, is rigid when it is uniquely determined by its local monodromies. Similarly, an $\ell$-adic representation over $\mathbb{P}^{1} \backslash S$ over a finite field is rigid if it is uniquely determined by its restriction to the Laurent power series fields at the singularities. N. Katz defines those concepts in [12] and shows a fundamental classification theorem: irreducible rigid local systems of tame $\ell$ adic representations are all obtained from rank one $\ell$-adic sheaves by applying Fourier transform and convolution ([12], Main Theorem 5.2.1). In fact the result is a little weaker as he shows the theorem under the assumption of cohomological rigidity but does not show the equivalence of the two notions of rigidity (see [12], Theorem 5.0.2). A similar result holds for complex local systems. One of Katz's key ideas is to define an index of rigidity [12] Chapter 3, and to show that under a suitable assumption on the system or $\ell$-adic representation, this index is invariant under Fourier transform. His proof relies on a theorem of Laumon on the local Fourier transform and the equivalence of categories it yields between local $\ell$-adic representations at $t=0$ and at $t^{\prime}=\infty$ with slopes $<1$ and between local $\ell$-adic reprenstations at $\infty$ with slopes $>1$ ([12], Theorem 3.0.2). Katz raises the question (op. cit, p. 10) whether an analogous invariance under Fourier transform is true for the index of rigidity of a meromorphic connection on $\mathbb{P}^{1}$. We show using the local Fourier transforms and Katz's arguments that this is the case (see Theorem 4.3). As an application we show that an irreducible meromorphic connection is rigid if and only if it has index of rigidity 2 . This characterization is not known for $\ell$-adic representations. This and the Fourier invariance of

\footnotetext{
*Received January 16, 2004; accepted for publication March 19, 2004.

${ }^{\dagger}$ Department of Mathematics, University of Chicago, Chicago, IL 60637, USA (bloch@ math.uchicago.edu).

${ }_{\ddagger}^{\ddagger}$ Mathematik, Universität Duisburg-Essen, FB6, Mathematik, 45117 Essen, Germany (esnault@ uni-essen.de).
} 
the index of rigidity yield a large class of rigid connections (4.9).

One may hope to use these ideas to classify rigid meromorphic connections with irregular singular points, though this question is not addressed here.

To be precise, the notion of a connection $\nabla$ on a sheaf $E$ or a module $M$ will refer to an integrable connection on a locally free sheaf or module of finite type. A meromorphic connection is permitted to have poles of finite order along a divisor at infinity. The usage here is a bit abusive in the sense that the polar structure is usually not part of the data. For example, the basic object of interest for us will be meromorphic connections on $\operatorname{Spec} k[[z]]$ (resp. on $\mathbb{P}^{1}$ ), by which we mean simply connections on Spec $k((z))$ (resp. $\mathbb{P}^{1}-S$ for some finite set $S$ ). Our connections are always taken to be algebraic, so a meromorphic extension exists. Occasionally, we will need to fix an extension of a meromorphic connection to a holonomic $\mathcal{D}$-module on $\mathbb{P}^{1}$ or Spec $k[[t]]$. For the theory of holonomic $\mathcal{D}$-modules, cf. [4] or [15]. In dimension 1 , a holonomic $\mathcal{D}$-module is simply a finitely-generated, torsion $\mathcal{D}$-module.

We remark that in [17], Malgrange constructs a complex analytic microlocalization for analytic connections which yields a microanalytic construction of $\mathcal{F}(0, \infty)$ and $\mathcal{F}(\infty, 0)$. His microlocal ideas were extended by R. Garcia Lopez to yield another construction of the local Fourier transform [7]. Our own work and that in op. cit. were done independently. We thank the author for sending us his paper.

Acknowledgements. We have greatly benefited from correspondence with Nick Katz on his theorem. We also thank Alexander Beilinson for helpful comments and correspondence, and Jean-Pierre Serre for correcting an error in the proof of Lemma 3.3 .

\section{Grothendieck's theorem on formal cohomology.}

TheOREm 2.1 ([8], Théorème 4.1.5)). : Let $f: X \rightarrow S$ be a proper morphism of noetherian schemes, let $S^{\prime} \subset S$ be a closed subset defined by the ideal sheaf $\mathcal{I} \subset \mathcal{O}_{S}$, and let $X^{\prime}=f^{-1}\left(S^{\prime}\right)$. Then if $\mathcal{F}$ is a coherent sheaf on $X$, the natural restriction map

$$
\varliminf_{\ell}^{\lim _{\ell}}\left(R^{n} f_{*} \mathcal{F}\right) \otimes_{\mathcal{O}_{S}} \mathcal{O}_{S} / \mathcal{I}^{\ell} \rightarrow \varliminf_{\ell}^{\lim _{\ell}} R^{n} f_{*}\left(\mathcal{F} \otimes_{\mathcal{O}_{X}} \mathcal{O}_{X} / f^{*} \mathcal{I}^{\ell}\right)
$$

is an isomorphism for all $n \geq 0$. Both sides coincide with the formal cohomology $R^{n} \widehat{f}_{*} \widehat{\mathcal{F}}$ on $\widehat{S}$, where $\widehat{-}$ refers to the formal scheme completions along $S^{\prime}$ and $f^{-1} S^{\prime}$.

We will apply this theorem to the following situation. Let $k$ be a field of characteristic 0 , and let $(M, \nabla)$ be a connection on $\mathbb{A}^{1} \backslash S$, where $S$ is a finite collection of points. We set $j: \mathbb{A}^{1} \backslash S \rightarrow \mathbb{A}^{1}, k: \mathbb{A}^{1} \rightarrow \mathbb{P}^{1}$ for the open embeddings. We consider the projection $p_{2}: X=\mathbb{P}^{1} \times \mathbb{P}^{1} \rightarrow \mathbb{P}^{1}$ and to distinguish the two factors, we denote by $t$ the parameter on the left $\mathbb{A}^{1}$ and by $t^{\prime}$ the parameter on the right $\mathbb{A}^{1}$. We consider the rank 1 connection $\psi=\left(\mathcal{O}_{\mathbb{A}^{1} \times \mathbb{A}^{1}}, d+d\left(t t^{\prime}\right)\right)$ on $\mathbb{A}^{1} \times \mathbb{A}^{1}$ and consider the cohomology

$$
R^{1} p_{2 *}\left(p_{1}^{*} k_{*} j_{*} M \stackrel{p_{1}^{*} \nabla \otimes \psi}{\longrightarrow} p_{1}^{*}\left(\omega(* T) \otimes k_{*} j_{*} M\right)\right),
$$

with $T=S \cup \infty$. In computing this cohomology, $\psi$ is viewed as a relative connection with operator $d_{t}+t^{\prime} d t$ which is extended meromorphically to $t=\infty$.

Write $\Omega$ for the direct image of $\Omega_{\mathbb{A}^{1} \times \mathbb{A}^{1}}^{1}$ on $\mathbb{P}^{1} \times \mathbb{P}^{1}$. The standard diagram, where the middle column calculates the de Rham cohomology on $\mathbb{P}^{1} \times \mathbb{P}^{1}$, (for which 
$\psi$ is viewed as a connection relative to $k$ ), and the right hand column is de Rham cohomology relative to $p_{2}$

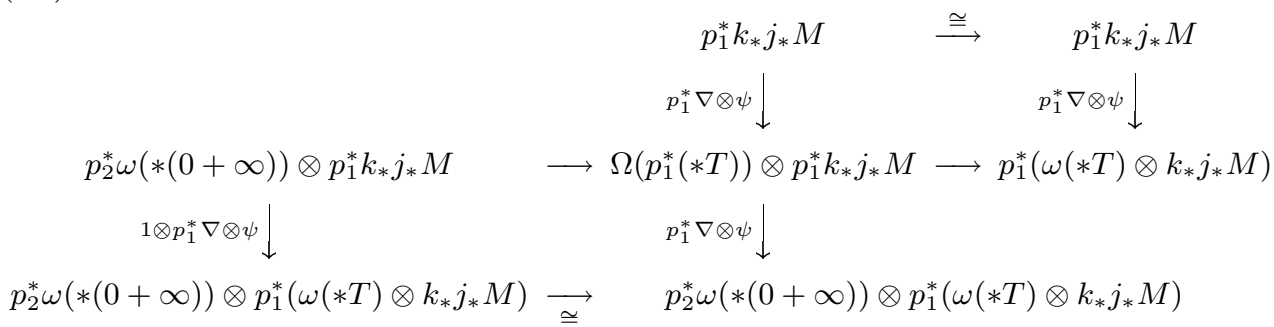

yields, via the connecting homomorphism, a connection

$$
(2.1) \rightarrow \omega(*(0+\infty)) \otimes(2.1) .
$$

We want to study the formal completion of this connection (which is the Fourier transform of $M$, see section 3 ) at the points $t^{\prime}=\infty$ and $t^{\prime}=0$.

Corollary 2.2. Assume given on $\mathbb{P}^{1}$ vector bundles $\mathcal{V}_{i} \subset k_{*} j_{*} M$ with the property that $\left(p_{1}^{*} \nabla \otimes \psi\right)\left(p_{1}^{*} \mathcal{V}_{1}\right) \subset p_{1}^{*} \omega(T) \otimes p_{1}^{*} \mathcal{V}_{2}$ so that the inclusion of complexes

$$
\begin{gathered}
\left(p_{1}^{*} \mathcal{V}_{1} \stackrel{p_{1}^{*} \nabla \otimes \psi}{\longrightarrow} p_{1}^{*} \omega(T) \otimes p_{1}^{*} \mathcal{V}_{2}\right) \subset \\
\left(p_{1}^{*} k_{*} j_{*} M \stackrel{p_{1}^{*} \nabla \otimes \psi}{\longrightarrow} p_{1}^{*}(\omega(* T)) \otimes k_{*} j_{*} M\right)
\end{gathered}
$$

is a quasi-isomorphism. Then one has

$$
\begin{gathered}
(2.1) \otimes_{\mathcal{O}_{\mathbb{P} 1}} k\left(\left(u^{\prime}\right)\right)= \\
\mathbb{H}^{1}\left(\mathbb{P}^{1}\left[\left[u^{\prime}\right]\right], \mathcal{V}_{1}\left[\left[u^{\prime}\right]\right] \stackrel{\nabla+t^{\prime} d t}{\longrightarrow}=\omega(T) \otimes \mathcal{V}_{2}\left[\left[u^{\prime}\right]\right]\right) \otimes_{k\left[\left[u^{\prime}\right]\right]} k\left(\left(u^{\prime}\right)\right)
\end{gathered}
$$

where $u^{\prime}=t^{\prime}-a$ for some point a or $u^{\prime}=\frac{1}{t^{\prime}}$. Here $\mathbb{P}^{1}\left[\left[u^{\prime}\right]\right]$ is the formal scheme obtained by completing $\mathbb{P}^{1} \times_{k}$ Spec $k\left[\left[u^{\prime}\right]\right]$ at the central fibre.

Proof. Indeed, the $E_{1}$ spectral sequence $E^{a b}=R^{b}\left(p_{2}\right)_{*} K^{a} \Rightarrow R^{a+b}\left(p_{2}\right)_{*} K^{\bullet}$ for the complex $K^{\bullet}: p_{1}^{*} \mathcal{V}_{1} \rightarrow p_{1}^{*} \omega(T) \otimes p_{1}^{*} \mathcal{V}_{2}$ yields

$$
(2.1) \otimes_{\mathcal{O}_{\mathbb{P}}} k\left[\left[u^{\prime}\right]\right]=\mathbb{H}^{1}\left(\mathbb{P}^{1}\left[\left[u^{\prime}\right]\right], \mathcal{V}_{1}\left[\left[u^{\prime}\right]\right] \stackrel{\nabla+t^{\prime} d t}{\longrightarrow} \omega(T) \otimes \mathcal{V}_{2}\left[\left[u^{\prime}\right]\right]\right)
$$

The assertion of the corollary follows by tensoring with $\otimes_{k\left[\left[u^{\prime}\right]\right]} k\left(\left(u^{\prime}\right)\right)$.

Corollary 2.3. Let $K=k\left(t^{\prime}\right)$. With the assumptions as in Corollary 2.2, one has

$$
\begin{gathered}
\mathbb{H}^{1}\left(\mathbb{P}^{1} \times_{k} K, p_{1}^{*} k_{*} j_{*} M \stackrel{p_{1}^{*} \nabla+\psi}{\longrightarrow} p_{1}^{*} \omega(T) \otimes k_{*} j_{*} M\right) \otimes_{K}=k\left(\left(u^{\prime}\right)\right)= \\
\mathbb{H}^{1}\left(\mathbb{P}^{1}\left[\left[u^{\prime}\right]\right], \mathcal{V}_{1}\left[\left[u^{\prime}\right]\right] \stackrel{\nabla+t^{\prime} d t}{\longrightarrow} \omega(T) \otimes \mathcal{V}_{2}\left[\left[u^{\prime}\right]\right]\right) \otimes_{k\left[\left[u^{\prime}\right]\right]} k\left(\left(u^{\prime}\right)\right)
\end{gathered}
$$

and the latter does not depend on the choice of $\mathcal{V}_{i}$ as in Corollary 2.2.

The aim of section 3 will be in particular to show the existence of such $\mathcal{V}_{i}$. 
In general, for a bounded below complex of sheaves $\mathcal{C}$ on a topological space $X$, one has a spectral sequence $E_{2}^{p, q}=H^{q}\left(X, \mathcal{H}^{p}\right) \Rightarrow \mathbb{H}^{p+q}(X, \mathcal{C})$ where $\mathcal{H}^{p}$ is the $p$-th cohomology sheaf of $\mathcal{C}$. We can apply this with $X$ the formal scheme $\mathbb{P}^{1}\left[\left[z^{\prime}\right]\right]$ with $z^{\prime}=\frac{1}{t^{\prime}}$, and $\mathcal{C}$ the complex

$$
\mathcal{V}_{1}\left[\left[z^{\prime}\right]\right] \stackrel{z^{\prime} \nabla+d t}{\longrightarrow} \omega(T) \otimes \mathcal{V}_{2}\left[\left[z^{\prime}\right]\right]
$$

as above, placed in degrees $[0,1]$. In this case, the differential is easily seen to be injective, so the hypercohomology in degree 1 is given by $H^{0}\left(\mathbb{P}^{1}\left[\left[z^{\prime}\right]\right], \mathcal{H}^{1}\right)$.

LEMma 2.4. The sheaf $\mathcal{H}^{1}$ in this case is supported at the points of $\mathbb{P}_{k}^{1}=\mathbb{P}^{1}\left[\left[z^{\prime}\right]\right]$ where $\nabla$ has singularities. At a point $s \in S \subset \mathbb{A}^{1}$ where $M$ has irregularity $n, \mathcal{H}_{s}^{1}$ is a free $k\left[\left[z^{\prime}\right]\right]$-module of rank $\operatorname{rank} M+n$. If $M$ is smooth or has a regular singular point at $\infty$, then $\mathcal{H}_{\infty}^{1}=(0)$.

$U$

Proof. For $x \in \mathbb{A}^{1}$ any point at finite distance, choose a $k$-vector space complement

$$
\left(\omega(T) \otimes \mathcal{V}_{2}\right)_{x}=\left(\mathcal{V}_{1, x} \wedge d t\right) \oplus U
$$

It is straightforward to identify $\mathcal{H}_{x}^{1} \cong U\left[\left[z^{\prime}\right]\right]$. The assertions for $x$ now follow from Deligne's theory of good lattices ([5], lemme 6.21). In particular, $U$ is a finite dimensional $k$-vector space of dimension $\operatorname{dim} \frac{1}{u} \mathcal{V}_{2} / \mathcal{V}_{2}+\operatorname{dim} \mathcal{V}_{2} / \mathcal{V}_{1}=\operatorname{rank}(M)+n$ (see the discussion in section 3, particularly formula (3.2) and Proposition 3.14, (i).) When $M$ has at worst a regular singular point at $\infty$, one can take $\mathcal{V}_{2, \infty}=\mathcal{V}_{1, \infty}(\infty)$. Since $\infty \in T$, in this case $\mathcal{V}_{1, \infty} \wedge d t=\left(\omega(T) \otimes \mathcal{V}_{2}\right)_{\infty}$, and one concludes by a variant of the above argument.

Let $x \in \mathbb{A}^{1}$ be as above, and write $\widehat{\mathcal{V}}_{i, x}=\mathcal{V}_{i, x} \otimes \widehat{\mathcal{O}}_{x}$ for the formal completion. We have

$$
\left(\omega(T) \otimes \mathcal{V}_{2}\right)_{x} / \mathcal{V}_{1, x} \wedge d t \cong\left(\omega(T) \otimes \widehat{\mathcal{V}}_{2}\right)_{x} / \widehat{\mathcal{V}}_{1, x} \wedge d t
$$

We conclude

COROLlary 2.5. With notation as above

$$
\mathcal{H}_{x}^{1} \cong=\operatorname{coker}\left(\widehat{\mathcal{V}}_{1, \mathrm{x}}\left[\left[\mathrm{z}^{\prime}\right]\right] \stackrel{\mathrm{z}^{\prime} \nabla+\mathrm{dt}}{\longrightarrow} \omega(\mathrm{T}) \otimes \widehat{\mathcal{V}}_{2, \mathrm{x}}\left[\left[\mathrm{z}^{\prime}\right]\right]\right) .
$$

Moreover, $\mathcal{H}_{x}^{1} \otimes_{k\left[\left[z^{\prime}\right]\right]} k\left(\left(z^{\prime}\right)\right)$ depends only on the formal meromorphic connection $M \otimes$ $k\left(\left(t_{x}\right)\right)$, where $t_{x}$ is a local parameter at $x$.

Proof. The last assertion follows from Corollary $2.3\left(\right.$ with $\left.u^{\prime}=z^{\prime}\right)$ and the spectral sequence.

REMARK 2.6. Let $x \neq 0$. Let us consider the Fourier transform of $M$ "centered at" $x$, that is consider the definition (2.5) with $t$ replaced by $t_{x}$. Call $\mathcal{H}^{1}\left(t_{x}\right)$ the hypercohomology sheaf. Then one obviously has

$$
\mathcal{H}^{1}\left(t_{x}\right)_{0} \otimes x d\left(\frac{1}{z^{\prime}}\right) \mathcal{H}_{x}^{1}
$$

$=$ where $x d\left(\frac{1}{z^{\prime}}\right)$ is the connection on $k\left(\left(z^{\prime}\right)\right)$ which to 1 assigns $x d\left(\frac{1}{z^{\prime}}\right)$. 
3. Local Fourier transforms. In [14], section 2, G. Laumon defines the local Fourier transforms of an $\ell$-adic representation over $\operatorname{Spec} \mathbb{F}_{q}((t))$. The aim of this section is to define the corresponding notion for connections on Spec $k((t))$, where $k$ is a field of characteristic 0 .

Let $k$ be a field of characteristic zero, and let $\mathcal{M}$ be a holonomic $\mathcal{D}=k\left[t, \partial_{t}\right]$ module over the affine line $\mathbb{A}^{1}=\operatorname{Spec} k[t]$. We recall the definition (see [11], or [15] chapter VI)

Definition 3.1. The Fourier transform $\mathcal{F}(\mathcal{M})$ of $\mathcal{M}$ is the $\mathcal{D}^{\prime}=k\left[t^{\prime}, \partial_{t^{\prime}}\right]$-module obtained by keeping the same $k$-vector space $\mathcal{M}$ but setting

i) $t^{\prime} \cdot m=-\partial_{t} \cdot m$

ii) $\partial_{t^{\prime}} \cdot m=t \cdot m$ for all $m \in \mathcal{M}$.

Lemma 3.2. $\mathcal{F}(\mathcal{M})$ is the Gauß-Manin connection on

$$
H^{1}\left(\mathcal{M} \otimes_{k} k\left[t^{\prime}\right] \stackrel{\partial_{t}+t^{\prime}}{\longrightarrow} \mathcal{M} \otimes_{k} k\left[t^{\prime}\right]\right) .
$$

Proof. One has the diagram

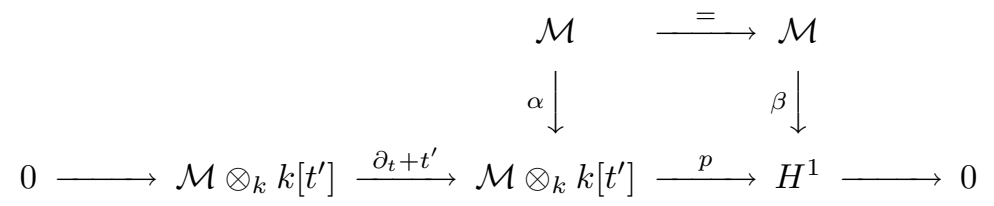

where $p$ is the quotient map to the first cohomology of $\partial_{t}+t^{\prime}, \alpha(m)=m \otimes 1, \beta=p \circ \alpha$. We first note that $\beta$ is an isomorphism of $k$-vector spaces. Indeed, $\beta$ is injective as $m \otimes 1$ can't be in the image of $\partial_{t}+t^{\prime}$. Given $\mu=\sum_{i=0}^{N} m_{i}\left(t^{\prime}\right)^{i}, m_{N} \neq 0, N \geq 1$, then $\mu-\left(\partial_{t}+t^{\prime}\right)\left(m_{N}\left(t^{\prime}\right)^{N-1}\right)$ has degree $\leq(N-1)$ in $t^{\prime}$. Inductively, one sees that every class in $H^{1}$ is the class of some $m \otimes 1$ so $\beta$ is onto. Now $p\left(m \otimes t^{\prime}\right)=-p\left(\partial_{t} m \otimes 1\right)$ which shows the relation i) of Definition 3.1. To see ii), one computes the Gauß-Manin connection on $p(m \otimes 1)$. One has $\left(\left(\nabla+d\left(t t^{\prime}\right)\right) / d t\right)(m \otimes 1)=\left(\partial_{t}+t^{\prime}\right)(m \otimes 1)+(t m \otimes$ 1) $\left.d t^{\prime}\right) \equiv(t m \otimes 1) d t^{\prime}$, implying ii).

To calculate Fourier transforms we will use Deligne's good lattices for irregular connections, defined in [5], Lemme 6.21. Let $X / k$ be a smooth curve, $j: X \subset$ $\bar{X}, \Sigma:=\bar{X} \backslash X$ be a smooth compactification, and let $\mathcal{M}$ be a smooth connection on $X$, meromorphic along $\Sigma$. By definition, a pair of good lattices $\mathcal{V}, \mathcal{W} \subset j_{*} \mathcal{M}$ is a pair of vector bundles on $\bar{X}$ satisfying the following conditions

1) $\mathcal{V} \subset \mathcal{W} \subset j_{*} \mathcal{M}$

2) $\nabla(\mathcal{V}) \subset \omega_{\bar{X}}(\Sigma) \otimes \mathcal{W}$

3) For any effective divisor $D$ supported on $\Sigma$, the inclusion of complexes

$$
\left(\mathcal{V} \stackrel{\nabla}{\longrightarrow} \omega_{\bar{X}}(\Sigma) \otimes \mathcal{W}\right) \rightarrow\left(\mathcal{V}(D) \stackrel{\nabla}{\longrightarrow} \omega_{\bar{X}}(\Sigma) \otimes \mathcal{W}(D)\right)
$$

is a quasi-isomorphism. In particular,

$$
\left(\mathcal{V} \stackrel{\nabla}{\longrightarrow} \omega_{\bar{X}}(\Sigma) \otimes \mathcal{W}\right) \rightarrow\left(j_{*} \mathcal{M} \stackrel{\nabla}{\longrightarrow} \omega_{\bar{X}} \otimes j_{*} \mathcal{M}\right)
$$

is a quasi-isomorphism. 
Notice that these conditions are purely local. For $\sigma \in \Sigma$, let $t_{\sigma}$ be a local parameter at $\sigma$. It suffices to construct lattices for $\mathcal{M} \otimes k\left(\left(t_{\sigma}\right)\right)$ satisfying the analogous conditions.

Deligne ([5], p.110-112) shows the existence of good lattices. If $\mathcal{V}, \mathcal{W}$ are good lattices, so are $\mathcal{V}(D), \mathcal{W}(D)$ for any divisor $D$ supported on $\Sigma$. At a point $\sigma \in \Sigma$ which is regular singular, one has $\mathcal{V} \otimes \mathcal{O}_{\bar{X}, \sigma}=\mathcal{W} \otimes \mathcal{O}_{\bar{X}, \sigma}$.

For $\sigma \in \Sigma$, the dimension of the finite dimensional $k$-vector space

$$
\mathcal{W} \otimes \mathcal{O}_{\bar{X}, \sigma} / \mathcal{V} \otimes \mathcal{O}_{\bar{X}, \sigma}
$$

is independent of the choice of $\mathcal{V}, \mathcal{W}$ and is equal to the irregularity of $\mathcal{M}$ at $\sigma$.

Lemma 3.3. Let $M$ be a connection on $k((t))$. Then the slopes of $M$ are $\leq 1$ (resp. $\geq 1)$ if and only if there exists a pair $\mathcal{V}, \mathcal{W}$ of good lattices such that $\mathcal{V} \subset \mathcal{W} \subset \mathcal{V}(0)$ (resp. $\mathcal{V} \subset \mathcal{V}(0) \subset \mathcal{W})$. Moreover for such a pair of good lattices, $\mathcal{V}(0)=\mathcal{W}$ if and only if the slopes are $=1$.

Proof. We may assume $M$ is indecomposable, i.e. not of the form $M_{1} \oplus M_{2}$ for $M_{i} \neq 0$. It follows ([15], Th. 1.5, p. 45) that $M$ has a single slope. The if part is clear. We prove necessity. If the slope is zero, the connection is regular singular. Then one has Deligne's lattices $\mathcal{V}$ ([5], Théorème 4.1 and Corollaire 3.14) with respect to which the connection has logarithmic poles thus $\mathcal{V}=\mathcal{W}$. Assume the slope is $>0$. As in [2], section 5.9, we may assume $M=N \otimes U$, where $U$ is regular singular with unipotent monodromy and $N=\pi_{*} L$ where $L$ is a rank 1 connection on a finite covering $\pi: \operatorname{Spec} K \rightarrow \operatorname{Spec} k((t))$. The integral closure of $k[[t]]$ in $K$ is a complete, equicharacteristic 0 discrete valuation ring, so it has the form $k^{\prime}[[u]]$ for some $k^{\prime} / k$ Galois finite ([19], Cor. 2, p. 280). Here $u$ satisfies an Eisenstein polynomial of some degree $p$ over $k^{\prime}((t))$. If we fix a trivialization $L=k^{\prime}((u)) \cdot e$ and write $\nabla(e)=e \otimes\left(a_{-n} u^{-n}+a_{-n+1} u^{-n+1}+\ldots\right) \frac{d u}{u}$ with $n \geq 1$, then we get a good lattice pair for $L$ taking $\mathcal{V}_{L}=k^{\prime}[[u]] \cdot e$ and $\mathcal{W}_{L}=u^{-n} \mathcal{V}_{L}$. Let $\pi^{\prime}: k^{\prime}((t)) \rightarrow k^{\prime}((u)), \sigma: k((t)) \rightarrow k^{\prime}((t))$. It is now straightforward to check that for $g \in \operatorname{Gal}\left(k^{\prime} / k\right)$,

$$
\left(g \pi_{*}^{\prime} \mathcal{V}_{L} \otimes \sigma^{*} U, g \pi_{*}^{\prime} \mathcal{W}_{L} \otimes \sigma^{*} U\right)
$$

is a good lattice pair for $\sigma^{*} M$, and that $M$ has slope $\frac{n}{p}$. We claim $\left(\left(\sum_{g \in \operatorname{Gal}\left(k^{\prime} / k\right)}\left(g \pi_{*}^{\prime} \mathcal{V}_{L}\right)\right) \otimes U,\left(\sum_{g \in \operatorname{Gal}\left(k^{\prime} / k\right)} g \pi_{*}^{\prime} \mathcal{W}_{L}\right) \otimes U\right)$ is a good lattice pair for $M$. To see this, note the connection has $H^{0}=H^{1}=(0)$. Write $A \rightarrow B \frac{d t}{t}$ for the complex associated to this lattice pair. We need to show the map

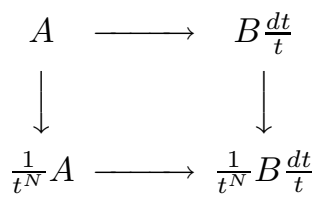

is a quasi-isomorphism. It is clear that both rows have $H^{0}=(0)$. For $H^{1}$, note that the bottom row is a quotient complex of the direct sum of $\frac{1}{t^{N}}$ times complexes associated to lattice pairs (3.3) over $g \in \operatorname{Gal}\left(k^{\prime} / k\right)$. Since this direct sum complex has $H^{1}=(0)$, it follows that both rows of (3.4) have $H^{1}=(0)$ as well.

When $\frac{n}{p} \leq 1$ (resp. $\frac{n}{p} \geq 1$ ) we have $\mathcal{W}_{L}=u^{-n} \mathcal{V}_{L} \subset t^{-1} \mathcal{V}_{L}\left(\right.$ resp. $\mathcal{W}_{L}=u^{-n} \mathcal{V}_{L} \supset$ $t^{-1} \mathcal{V}_{L}$ ). The assertion of the lemma follows by applying $\pi_{*}^{\prime}$, saturating for $k^{\prime} / k$ and tensoring with $U$. 
REMARK 3.4. It is not true that if the slope condition is as in Lemma 3.3, then all pairs of good lattices fulfill the relations of the lemma. Indeed, the ones constructed by Deligne [5], Lemme 6.21 do not always. For example, the connection on $\oplus_{1}^{3} \mathcal{O}$ with connection matrix

$$
\left(\begin{array}{ccc}
0 & 0 & \frac{1}{t^{2}} \\
1 & 0 & 0 \\
0 & 1 & 0
\end{array}\right) \frac{d t}{t}
$$

does not. The slope is $\frac{2}{3}<1, \mathcal{V}$ as a $\mathcal{O}$-module is generated by $e_{1}, e_{2}, e_{3}$ while $\mathcal{W}$ is generated by $e_{2}, e_{3}, \frac{1}{t^{2}} e_{1}$.

Let $M$ be a connection on $k((t))$. Recall from [10] Theorem (2.4.10) that there is a canonical (but not unique) functorial smooth extension $\mathcal{M}$ to $\mathbb{G}_{m}=\operatorname{Spec} k\left[t, t^{-1}\right]$ which has a regular singular point at $t=\infty$. We shall refer to $\mathcal{M}$ as the Katz extension of $M$. Our arguments will use the existence of a Katz extension, but nothing about its properties.

Proposition-Definition 3.5 (Local Fourier from 0 to $\infty$ ). Let $M$ be a connection on $k((t))$ and let $\mathcal{M}$ be the Katz extension to a meromorphic connection on $\mathbb{P}^{1}$ with regular singular point at $t=\infty$. Let $t^{\prime}$ be the Fourier transform coordinate, and write $z^{\prime}=\frac{1}{t^{\prime}}$. Then the Fourier transform connection (Definition 3.1) restricted to the Laurent series field at $t^{\prime}=\infty, \mathcal{F}(\mathcal{M}) \otimes_{k\left[t^{\prime}\right]} k\left(\left(z^{\prime}\right)\right)$ on $k\left(\left(z^{\prime}\right)\right)$ depends only on $M$ and not on the choice of $\mathcal{M}$. We call it the local Fourier transform of $M$ from 0 to $\infty$ and denote it by $\mathcal{F}(0, \infty)(M)$. Concretely, if $\widehat{\mathcal{V}}, \widehat{\mathcal{W}}$ is a good lattice pair for the formal connection $M$,

$$
\mathcal{F}(0, \infty)(M)=\operatorname{coker}\left(\widehat{\mathcal{V}}\left(\left(\mathrm{z}^{\prime}\right)\right) \stackrel{\mathrm{z}^{\prime} \partial_{\mathrm{t}}+1}{\longrightarrow} \frac{1}{\mathrm{t}} \widehat{\mathcal{W}}\left(\left(\mathrm{z}^{\prime}\right)\right)\right) .
$$

Proof. We apply the discussion of section 2 to the Katz extension $\mathcal{M}$ of $M$. Thus, with notation as in Corollary $2.5, \mathcal{F}(0, \infty)(M):=\mathcal{H}_{0}^{1} \otimes k\left(\left(z^{\prime}\right)\right)$. Independence of choice of good lattices follows from that corollary.

REMARK 3.6. If the connection $M$ on Spec $k((t))$ extends smoothly across Spec $k[[t]], \mathcal{F}(\mathcal{M})$ is supported at $t^{\prime}=0$ and $\mathcal{F}(0, \infty)(M)=(0)$. For this reason, we will assume when working with the local Fourier transform that $M^{\nabla}=(0)$.

The construction of $\mathcal{F}(0, \infty)(M)$ is independent of the choice of a good lattice pair. In particular, we can take $\widehat{\mathcal{W}}$ as large as we like. The composition

$$
\frac{1}{t} \widehat{\mathcal{W}} \hookrightarrow \frac{1}{t} \widehat{\mathcal{W}}\left(\left(z^{\prime}\right)\right) \rightarrow \mathcal{F}(0, \infty)(M)
$$

therefore extends to a $k$-linear map

$$
\iota: M \rightarrow \mathcal{F}(0, \infty)(M) .
$$

Proposition 3.7. Assume $M^{\nabla}=(0)$. Then $\iota$ is an isomorphism of $k$-vector spaces. One has $\iota \circ \partial_{t}=-\frac{1}{z^{\prime}} \circ \iota$ and $\iota \circ t=-\left(z^{\prime}\right)^{2} \partial_{z^{\prime}} \circ \iota$.

Proof. Suppose $\iota(m)=0$. Taking $\widehat{\mathcal{W}}$ to be large, we can assume $m \in \frac{1}{t} \widehat{\mathcal{W}}$. Then $m$ has to be of the shape $\left(z^{\prime} \partial_{t}+1\right)\left(\sum_{\ell=N}^{\infty}\left(z^{\prime}\right)^{\ell} v_{\ell}\right)=\left(z^{\prime}\right)^{N} v_{N}+\sum_{\ell \geq N+1}\left(z^{\prime}\right)^{\ell}\left(\partial_{t} v_{\ell-1}+v_{\ell}\right)$ 
for some $v_{\ell} \in \widehat{\mathcal{V}}$. This implies $N=0,\left(\partial_{t}\right)^{\ell} m=(-1)^{\ell} v_{\ell} \in \widehat{\mathcal{V}}$ for $\ell \geq 0$ so

$$
m=\left(z^{\prime} \partial_{t}+1\right)\left(\sum_{\ell=0}^{\infty}(-1)^{\ell} z^{\ell \ell} \partial_{t}^{\ell} m\right) .
$$

But the fact that $\left(\partial_{t}\right)^{\ell} m \in \widehat{\mathcal{V}}$ means the $k[[t]]\left[\partial_{t}\right]$-submodule of $M$ generated by $m$ is finitely generated as a $k[[t]]$-module and so necessarily has a horizontal section, contradicting our assumption.

The assertion that $\iota \circ \partial_{t}=-\frac{1}{z^{\prime}} \circ \iota$ is clear. Our assumption that $M^{\nabla}=(0)$ implies $\partial_{t}: M \cong M$ (cf. for example [16], Thm. $2.1(\mathrm{~b})$ ), so we may speak of $\partial_{t}^{-1}$. This operator is $t$-adically contracting. Indeed, $\widehat{\mathcal{V}} \subset \widehat{\mathcal{W}}$ and $\partial_{t}: \widehat{\mathcal{V}} \cong \frac{1}{t} \widehat{\mathcal{W}}$. Clearly $\iota \circ \partial_{t}^{-1}=-z^{\prime} \circ \iota$. It follows that the image of $\iota$ is closed under taking Laurent series in $z^{\prime}$, from which surjectivity is clear. As for $\iota \circ t=-\left(z^{\prime}\right)^{2} \partial_{z^{\prime}} \circ \iota$, the computation is as in Lemma 3.2.

Definition 3.8. [Local Fourier from $\infty$ to 0 ] Let $M$ be a connection on $k((z))$. Assume $M^{\nabla}=(0)$ and that the slopes ([15], chap. III) are all $<1$. Let $\widehat{\mathcal{V}}, \widehat{\mathcal{W}}$ be a pair of good lattices for $M$. By Lemma 3.3 we may assume $z^{2} \partial_{z} \widehat{\mathcal{V}} \subset \widehat{\mathcal{V}}$. Then

$$
\mathcal{F}(\infty, 0)(M):=\operatorname{coker}\left(\widehat{\mathcal{V}}\left(\left(t^{\prime}\right)\right) \stackrel{-z^{2} \partial_{z}+t^{\prime}}{\longrightarrow} \widehat{\mathcal{V}}\left(\left(t^{\prime}\right)\right)\right) .
$$

Proposition 3.9. Let $M$ be as in the definition. Then $\mathcal{F}(\infty, 0)(M)$ is independent of the choice of good lattices. The natural map

$$
\iota: \widehat{\mathcal{V}} \hookrightarrow \widehat{\mathcal{V}}\left(\left(t^{\prime}\right)\right) \rightarrow \mathcal{F}(\infty, 0)(M)
$$

extends to an isomorphism of $k$-vector spaces $\iota: M \cong \mathcal{F}(\infty, 0)(M)$. We have $\iota \circ z^{2} \partial_{z}=$ $t^{\prime} \circ \iota$ and $\iota \circ \frac{1}{z}=-\partial_{t^{\prime}} \circ \iota$

Proof. To show independence of the choice of lattices, let $\widehat{\mathcal{V}}, \widehat{\mathcal{W}}$ and $\widehat{\mathcal{V}}^{\prime}, \widehat{\mathcal{W}}^{\prime}$ be two pairs of good lattices with $\widehat{\mathcal{V}}, \widehat{\mathcal{V}}^{\prime}$ stable under $z^{2} \partial_{z}$. We may assume $\widehat{\mathcal{V}} \subset \widehat{\mathcal{V}}^{\prime}$, and we have to show $-z^{2} \partial_{z}+t^{\prime}$ is an isomorphism on $\left(\widehat{\mathcal{V}}^{\prime} / \widehat{\mathcal{V}}\right)\left(\left(t^{\prime}\right)\right)$. But this is clear because the slope condition forces $z^{2} \partial_{z}$ to be nilpotent.

Because $z^{2} \partial_{z}$ is injective on $M$, an equation of the form

$$
m=\left(-z^{2} \partial_{z}+t^{\prime}\right) \sum_{n=N}^{\infty} v_{n} t^{\prime n}
$$

forces $m=-\left(z^{2} \partial_{z}\right)^{n+1} v_{n}, v_{n} \in \widehat{\mathcal{V}}$. Again by the slope condition, this forces $m=0$, so $M \hookrightarrow \mathcal{F}(\infty, 0)(M)$.

The identity $\iota \circ z^{2} \partial_{z}=t^{\prime} \circ \iota$ is clear from the definition. Since $z^{2} \partial_{z}$ is bijective and contracting, it follows that the image of $\iota$ is closed under taking Laurent series in $t^{\prime}$, so $\iota$ is surjective as well. The proof that $\iota \circ \frac{1}{z}=\partial_{t^{\prime}} \circ \iota$, is as in Lemma 3.2.

Proposition 3.10. The functors $\mathcal{F}(0, \infty)$ and $\mathcal{F}(\infty, 0)$ are inverses and define equivalences of categories

$$
\begin{aligned}
& \{k((t))-\text { connections with no horiz. sects. }\} \leftrightarrow \\
& \left\{k\left(\left(z^{\prime}\right)\right)-\text { connections with no horiz. sects. and slopes }<1\right\}
\end{aligned}
$$


Proof. Let $M$ be a $k((t))$-connection with $M^{\nabla}=(0)$. By Proposition 3.7, $M \cong \mathcal{F}(0, \infty)(M)$ and the action of $-z^{\prime 2} \partial_{z^{\prime}}$ on $\mathcal{F}(0, \infty)(M)$ corresponds to multiplication by $t$ on $M$. In particular, $\mathcal{F}(0, \infty)(M)^{\nabla}=(0)$. Similarly, given $N$ a $k\left(\left(z^{\prime}\right)\right)$-connection with no horizontal sections and slopes $<1$, we have by Proposition $3.9, N \cong \mathcal{F}(\infty, 0)(N)$ and $\partial_{t}$ on $\mathcal{F}(\infty, 0)(N)$ corresponds to multiplication by $-\frac{1}{z^{\prime}}$ on $N$, so $\mathcal{F}(\infty, 0)(N)$ has no global sections and the functors are defined. Finally, under the vector space identifications, the operators intertwine as indicated:

$$
\begin{array}{ccc} 
& M \cong \mathcal{F}(0, \infty)(M) \cong \mathcal{F}(\infty, 0) & (\mathcal{F}(0, \infty)(M)) \\
t & -z^{2} \partial_{z} & -t \\
\partial_{t} & -\frac{1}{z} & -\partial_{t}
\end{array}
$$

It follows that $\mathcal{F}(\infty, 0) \circ \mathcal{F}(0, \infty)=[t \mapsto-t]^{*}$. The argument in the other direction is similar.

Definition 3.11. Let $M$ be a connection on $k((z))$ and assume all slopes of $M$ are $>1$. Let $\widehat{\mathcal{V}}, \widehat{\mathcal{W}}$ be a good lattice pair with $\frac{1}{z} \widehat{\mathcal{V}} \subset \widehat{\mathcal{W}}$. Define

$$
\mathcal{F}(\infty, \infty)(M):=\operatorname{coker}\left(\widehat{\mathcal{V}}\left(\left(z^{\prime}\right)\right) \stackrel{-z^{\prime} z^{2} \partial_{z}+1}{\longrightarrow} z \widehat{\mathcal{W}}\left(\left(z^{\prime}\right)\right)\right) .
$$

$\mathcal{F}(\infty, \infty)(M)$ is a connection on $k\left(\left(z^{\prime}\right)\right)$.

Proposition 3.12. Let $M$ be a connection on $k((z))$ with slopes $>1$.

(i) $\mathcal{F}(\infty, \infty)(M)$ is independent of the choice of good lattice pair.

(ii) The evident projection $z \widehat{\mathcal{W}} \rightarrow \mathcal{F}(\infty, \infty)(M)$ extends to an isomorphism of $k$-vector spaces $\iota: M \cong \mathcal{F}(\infty, \infty)(M)$.

(iii) The operators $z^{2} \partial_{z}$ and $-\frac{1}{z}$ on $M$ coincide with the operators $\frac{1}{z^{\prime}}$ and $z^{\prime 2} \partial_{z^{\prime}}$ on $\mathcal{F}(\infty, \infty)(M)$. In particular, $\mathcal{F}(\infty, \infty)(M)$ has all slopes $>1$.

(iv) $\mathcal{F}(\infty, \infty) \circ \mathcal{F}(\infty, \infty)(M) \cong \sigma^{*} M$, where $\sigma: k((z)) \rightarrow k((z))$ is the automorphism $z \mapsto-z$. In particular, $\mathcal{F}(\infty, \infty)$ is an auto-equivalence of the category of connections on $k((z))$ with slopes $>1$.

(v) Let $\mathcal{M}$ be a Katz extension of $M$ to a meromorphic connection on $\mathbb{P}^{1}$, smooth over $\mathbb{G}_{m}$ with a regular singular point at $z=\infty$. Then $\mathcal{F}(\infty, \infty)(M) \cong$ $\mathcal{F}(\mathcal{M}) \otimes k\left(\left(z^{\prime}\right)\right)$.

Proof.

(i) It suffices to consider good lattice pairs $\widehat{\mathcal{V}}, \widehat{\mathcal{W}}$ and $\widehat{\mathcal{V}}^{\prime}, \widehat{\mathcal{W}}^{\prime}$ with $\widehat{\mathcal{V}} \subset \widehat{\mathcal{V}}^{\prime}$ and $\widehat{\mathcal{W}} \subset \widehat{\mathcal{W}}^{\prime}$. Again by [16], Thm. 2.1 (b), z $\partial_{z}: \widehat{\mathcal{V}} \cong \widehat{\mathcal{W}}\left(\right.$ resp. $\left.\widehat{\mathcal{V}}^{\prime} \cong \widehat{\mathcal{W}}^{\prime}\right)$. It follows easily that

$$
\left(\widehat{\mathcal{V}}^{\prime} / \widehat{\mathcal{V}}\right)\left(\left(z^{\prime}\right)\right) \stackrel{-z^{\prime} z^{2} \partial_{z}+1}{\longrightarrow} z\left(\widehat{\mathcal{W}}^{\prime} / \widehat{\mathcal{W}}\right)\left(\left(z^{\prime}\right)\right)
$$

is an isomorphism, proving (i).

(ii) The proof here is analogous to Propositions 3.7 and 3.9. The identity $z w=$ $\left(1-z^{\prime} z^{2} \partial_{z}\right)\left(\sum_{r>-N} v_{r} z^{\prime r}\right)$ with $v_{r} \in \widehat{\mathcal{V}}$ forces $v_{r}=0, r<0, v_{0}=z w$, and $v_{r}=z^{2} \partial_{z} v_{r-1}$. Since the slopes are all $>1$, this is a contradiction unless $w=0$. Thus $M \hookrightarrow \mathcal{F}(\infty, \infty)(M)$. Also $\left(z^{2} \partial_{z}\right)^{-1}$ is defined and $z$-adically contracting on $M$. Since this operator intertwines $z^{\prime}$ on $\mathcal{F}(\infty, \infty)(M)$, it is clear that the image of $M$ is stable under taking Laurent series in $z^{\prime}$, from which (ii) follows. 
(iii) and (iv) are straightforward from (ii).

(v) We can assume $\widehat{\mathcal{V}}$ and $\widehat{\mathcal{W}}$ come by completion at $z=0$ from a global good lattice pair $\mathcal{V}, \mathcal{W}$, and that these lattices have no higher cohomology, so

$$
\begin{aligned}
\mathcal{F}(\mathcal{M}) \otimes & k\left(\left(z^{\prime}\right)\right) \cong \\
& \operatorname{coker}\left(\Gamma\left(\mathbb{P}^{1}, \mathcal{V}\right) \otimes k\left(\left(z^{\prime}\right)\right) \stackrel{-z^{\prime} z^{2} \partial_{z}+1}{\longrightarrow} \Gamma\left(\mathbb{P}^{1}, z \mathcal{W}\right) \otimes k\left(\left(z^{\prime}\right)\right)\right) .
\end{aligned}
$$

Indeed, let $j: \mathbb{G}_{m} \hookrightarrow \mathbb{P}^{1}$. Consider the diagram

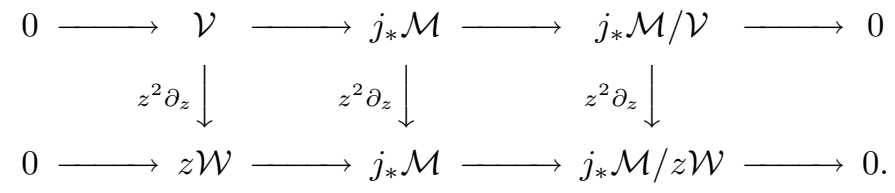

By definition of good lattice pair, the arrow on the right is an isomorphism. Let $p: j_{*} \mathcal{M} / \mathcal{V} \rightarrow j_{*} \mathcal{M} / z \mathcal{W}$ be the natural surjection. Then $z^{2} \partial_{z}-\frac{1}{z^{\prime}} p:$ $j_{*} \mathcal{M} / \mathcal{V}\left(\left(z^{\prime}\right)\right) \rightarrow j_{*} \mathcal{M} / z \mathcal{W}\left(\left(z^{\prime}\right)\right)$ is easily checked to be an isomorphism. The assertion in (3.17) follows by tensoring (3.18) with $k\left(\left(z^{\prime}\right)\right)$, replacing $z^{2} \partial_{z}$ with $z^{2} \partial_{z}-\frac{1}{z^{\prime}}$ in (3.18) and taking $R \Gamma$, using vanishing for $H^{1}$ on the left. It follows from (3.15) and (3.17) that there is a natural map $\mathcal{F}(\mathcal{M}) \otimes k\left(\left(z^{\prime}\right)\right) \rightarrow$ $\mathcal{F}(\infty, \infty)(M)$. To see injectivity, an identity of the form

$$
\sum_{i \geq m} w_{i} z^{i}=\left(1-z^{\prime} z^{2} \partial_{z}\right) \sum_{j \geq n} \hat{v}_{j} z^{j}
$$

with $w_{i} \in \Gamma\left(\mathbb{P}^{1}, z \mathcal{W}\right), \hat{v}_{j} \in \widehat{\mathcal{V}}, w_{m} \neq 0, \hat{v}_{n} \neq 0$ yields that $n=m$, and $w_{m}=\hat{v}_{m} \in \Gamma\left(\mathbb{P}^{1}, z \mathcal{W}\right) \cap \hat{\mathcal{V}}=\Gamma\left(\mathbb{P}^{1}, \mathcal{V}\right)$. Then, recursively $\hat{v}_{j}=w_{j}+z^{2} \partial_{z} \hat{v}_{j-1} \in$ $\widehat{\mathcal{V}} \cap \Gamma\left(\mathbb{P}^{1}, z \mathcal{W}\right)=\Gamma\left(\mathbb{P}^{1}, \mathcal{V}\right)$

For surjectivity, we assume moreover that $\mathcal{W}$ is so positive that $\Gamma\left(\mathbb{P}^{1}, z \mathcal{W}\right) \rightarrow$ $z \widehat{\mathcal{W}} / \widehat{\mathcal{V}}$. Given $\hat{w} \in z \widehat{\mathcal{W}}$, we can then find $w \in \Gamma\left(\mathbb{P}^{1}, z \mathcal{W}\right)$ with $\hat{v}:=\hat{w}-w \in \widehat{\mathcal{V}}$. Then

$$
\begin{aligned}
(\hat{w}-w) z^{\prime N}=\left(1-z^{\prime} z^{2} \partial_{z}\right) \hat{v} z^{\prime N}+z^{2} \partial_{z} \hat{v} z^{\prime N+1} \in & \\
& \left(1-z^{\prime} z^{2} \partial_{z}\right) \hat{\mathcal{V}} \otimes z^{\prime N} k\left[\left[z^{\prime}\right]\right]+z \widehat{\mathcal{W}} z^{\prime N+1}
\end{aligned}
$$

Iterating in this fashion, we get a convergent series in $z^{\prime}$.

Remark 3.13. One can extend Definition 3.11 to the case where the slopes of $M$ on $k((z))$ are $\leq 1$. However, when $M$ has slopes 1 , by Lemma 3.3 one can take $\widehat{\mathcal{V}}=z \widehat{\mathcal{W}}$ and $\mathcal{F}(\infty, \infty)(M)=0$.

Proposition 3.14. With notations and assumptions as above (in particular, $M^{\nabla}=(0)$ and the appropriate slope conditions are assumed to hold for $M$, cf. Propositions 3.5, 3.7, 3.12)), we have the following rank and irregularity relations for the local Fourier transforms:

(i) irreg. $\mathcal{F}(0, \infty)(\mathrm{M})=$ irreg.(M); rk $\mathcal{F}(0, \infty)(\mathrm{M})=\operatorname{rk}(\mathrm{M})+$ irreg.(M).

(ii) irreg. $\mathcal{F}(\infty, 0)(\mathrm{M})=$ irreg.(M); $\operatorname{rk} \mathcal{F}(\infty, 0)(\mathrm{M})=\operatorname{rk}(\mathrm{M})-$ irreg.(M).

(iii) irreg. $\mathcal{F}(\infty, \infty)(\mathrm{M})=$ irreg.(M); rk $\mathcal{F}(\infty, \infty)(\mathrm{M})=-$ rk $(\mathrm{M})+$ irreg.(M). 
Proof.

(i) Let $\widehat{\mathcal{V}}, \widehat{\mathcal{W}}$ be a good lattice pair for the connection $M$ over $k((t))$. We have (using [16], Thm. 2.1(b) and the properties of good lattices from section 3) $\partial_{t}: \widehat{\mathcal{V}} \cong \frac{1}{t} \widehat{\mathcal{W}}$. In particular, $\partial_{t}^{-1}$ stabilizes $\widehat{\mathcal{V}}$. Under the identification $\iota: M \cong \mathcal{F}(0, \infty)(M)$ (cf. Proposition 3.7), $\partial_{t}$ corresponds to multiplication by $z^{\prime-1}$, so $\iota \widehat{\mathcal{V}} \subset \mathcal{F}(0, \infty)(M)$ is a $k\left[\left[z^{\prime}\right]\right]$-submodule. Also, $z^{\prime} \partial_{z^{\prime}} \iota \widehat{\mathcal{V}}=\iota \partial_{t} t \widehat{\mathcal{V}}=$ $\iota\left(t \partial_{t}+1\right) \widehat{\mathcal{V}}$. Replacing $\widehat{\mathcal{V}}$ by $t^{N} \widehat{\mathcal{V}}$ for $N>>0$, we may assume finally that

$$
\widehat{\mathcal{W}}=\left(t \partial_{t}+1\right) \widehat{\mathcal{V}}
$$

Indeed, the formal connection $M$ splits, $M=M_{r} \oplus M_{i}$, into regular singular and irregular parts ([15], p. 51, Thm. 2.3). We may assume a similar decomposition for the lattice pair. To pass from the good lattice condition $\widehat{\mathcal{W}}=t \partial_{t} \widehat{\mathcal{V}}$ to (3.21) there is no difficulty in the irregular case because the slopes are $>0$. In the regular singular case, scaling the lattices with a large power of $t$ eliminates the eigenvalue -1 , so (3.21) holds in that case as well. We have, therefore $z^{\prime} \partial_{z^{\prime}} \widehat{\mathcal{V}}=\iota \widehat{\mathcal{W}}$, so $\iota \widehat{\mathcal{V}}, \iota \widehat{\mathcal{W}}$ are a good lattice pair for $\mathcal{F}(0, \infty)(M)$. In particular

$$
\text { irreg. }(\mathcal{F}(0, \infty)(\mathrm{M}))=\operatorname{dim}_{\mathrm{k}} \iota \widehat{\mathcal{W}} / \widehat{\mathcal{V}}=\operatorname{dim}_{\mathrm{k}} \widehat{\mathcal{W}} / \widehat{\mathcal{V}}=\operatorname{irreg} .(\mathrm{M}) \text {. }
$$

From Proposition-Definition 3.5, we have $\operatorname{rk} \mathcal{F}(0, \infty)(\mathrm{M})$ equals the generic rank of $\mathcal{F}(\mathcal{M})$ where $\mathcal{M}$ is the Katz extension coinciding with $M$ at 0 . A standard index calculation, using that the Fourier sheaf has irregularity 1 at $\infty$, yields irreg. $(\mathrm{M})+\operatorname{rk}(\mathrm{M})$, as claimed. A self-contained computation in the spirit of the article is to consider a finite dimensional $k$-vector space $U$ as in (2.6). It has dimension equal to $\frac{1}{t} \widehat{\mathcal{W}} / \widehat{\mathcal{V}}$, which is the rank of $\mathcal{F}(0, \infty)(M)$, that is $\operatorname{dim} \frac{1}{z} \widehat{\mathcal{W}} / \widehat{\mathcal{W}}+\operatorname{dim} \widehat{\mathcal{W}} / \widehat{\mathcal{V}}=\operatorname{rk}(M)+\operatorname{irreg} .(M)$.

(ii) Both assertions follow from (i) together with Proposition 3.10. The direct computation as above shows again that $\iota \widehat{\mathcal{V}}, \iota \widehat{\mathcal{W}}$ is a good lattice pair if $\widehat{\mathcal{V}}, \widehat{\mathcal{W}}$ is.

(iii) Here $M$ is a $k((z))$-connection, and we have a $k$-vector space isomorphism $\iota: M \cong \mathcal{F}(\infty, \infty)(M)$. We have

$$
z^{\prime} \iota \widehat{\mathcal{W}}=\iota\left(z^{2} \partial_{z}\right)^{-1} \widehat{\mathcal{W}} \subset \iota \widehat{\mathcal{W}}
$$

so $\iota \widehat{\mathcal{W}} \subset \mathcal{F}(\infty, \infty)(M)$ is a $k\left[\left[z^{\prime}\right]\right]$-lattice. Also $z \partial_{z}: \widehat{\mathcal{V}} \cong \widehat{\mathcal{W}}$ implies

$$
\begin{gathered}
\iota \widehat{\mathcal{V}}=\iota\left(z \partial_{z}\right)^{-1} \widehat{\mathcal{W}}=\iota\left(z^{-1} z^{2} \partial_{z}\right)^{-1} \widehat{\mathcal{W}}=z^{\prime}\left(z^{\prime 2} \partial_{z^{\prime}}\right)^{-1} \iota \widehat{\mathcal{W}} \\
\iota \widehat{\mathcal{W}}=\left(z^{\prime} \partial_{z^{\prime}}-1\right) \iota \widehat{\mathcal{V}} .
\end{gathered}
$$

Since in the case of $\mathcal{F}(\infty, \infty)$ the slopes are assumed $>1$, it follows that $\iota \widehat{\mathcal{W}}=z^{\prime} \partial_{z^{\prime}} \iota \widehat{\mathcal{V}}$ so $\iota \widehat{\mathcal{V}}, \iota \widehat{\mathcal{W}}$ are a good lattice pair for $\mathcal{F}(\infty, \infty)(M)$. It follows as in (i) that $M$ and $\mathcal{F}(\infty, \infty)(M)$ have the same irregularity.

Finally, we compute the rank directly. Consider

$$
\widehat{\mathcal{V}}\left[\left[z^{\prime}\right]\right] \stackrel{z^{\prime} \partial_{z}-1 / z^{2}}{\longrightarrow} \frac{1}{z} \widehat{\mathcal{W}}\left[\left[z^{\prime}\right]\right]
$$

Note $z^{-1} \widehat{\mathcal{W}} / z^{-2} \widehat{\mathcal{V}}$ is a $k$-vector space of $\operatorname{rank} \operatorname{dim} z^{-1} \widehat{\mathcal{W}} / z^{-1} \widehat{\mathcal{V}}-$ $\operatorname{dim} z^{-2} \widehat{\mathcal{V}} / z^{-1} \widehat{\mathcal{V}}=$ irreg. $(M)-\operatorname{rk}(M)$. Write $z^{-1} \widehat{\mathcal{W}}=S \oplus z^{-2} \widehat{\mathcal{V}}$ for a $k$ vector space $S$ of this rank. It is straightforward to check that $\mathcal{F}(\infty, \infty)(M) \cong$ $S\left(\left(z^{\prime}\right)\right)$ as a $k\left(\left(z^{\prime}\right)\right)$-module, so the rank is the same. 
4. Application to rigidity. The aim of this section is to apply the equivalence of categories proven in section 3 to the computation of the index of rigidity of the Fourier transform of holonomic $\mathcal{D}$ modules on $\mathbb{P}^{1}$.

Let us recall the notion of rigidity as defined by N. Katz ([12], Introduction). Let $U \subset \mathbb{P}^{1}$ be a non-empty open set, defined over a field $k$. If $k$ is a finite field and $M$ is a $\ell$-adic representation on $U$, then $M$ is said to be rigid if it is uniquely recognized by the induced local $\ell$-adic representations at the punctures $\mathbb{P}^{1} \backslash U$. If $k=\mathbb{C}$ and $M$ is a local system on $U$, then $M$ is said to be rigid if it is uniquely recognized by its local monodromies at the punctures. The main theorem proven by N. Katz in [12], Theorem 5.2.1 is that a rigid local system or a tame cohomologically rigid $\ell$-adic representation is always obtained from a rank 1 one after taking convolution and Fourier transform. An important technical tool to prove this fundamental classification theorem is the notion of index of rigidity and the fact that it is preserved by Fourier transforms [12], Theorem 3.0.2. With this, he is then able in the tame case to inductively lower the rank of the representation by a suitable rank one twist and convolution. He shows ([12], Theorem 5.0.2) that an irreducible cohomologically rigid $\ell$-adic prepresentation is rigid.

Our aim is to show that our construction of local Fourier transforms and the accompanying equivalences of categories implies invariance of the index of rigidity by Fourier transform in the $\mathcal{D}$-module case.

Let $X$ be a smooth, complete curve. Recall ([13], p. 65, Prop. (2.9.8)) that if $M$ is a connection (i.e. a smooth holonomic $\mathcal{D}$-module) on an open $\ell: U \hookrightarrow X$, then its middle extension $\ell_{! *} M$ sits in an exact sequence of $\mathcal{D}$ modules on $X$

$$
0 \rightarrow \ell_{!_{*}} M \rightarrow \ell_{*} M \rightarrow \oplus_{x \in X \backslash U}\left(i_{x}\right)_{*}\left[\left(\left(M^{\vee} \otimes \widehat{K}_{x}\right)^{\nabla}\right)^{\vee}\right] \rightarrow 0
$$

where $i_{x}:\{x\} \rightarrow X$ is the closed embedding, $i_{x *}$ is the $\mathcal{D}$-module direct image, and $\widehat{K}_{x}$ is the complete local field at $x$.

REMARK 4.1. The cokernel in (4.1) can be written

$$
\oplus_{x}\left(i_{x}\right)_{*} H_{D R}^{1}\left(\operatorname{Spec} \widehat{K}_{x}, M \otimes \widehat{K}_{x}\right) .
$$

In particular, taking cohomology yields

$$
0 \rightarrow H^{1}\left(X, \ell_{!_{*}} M\right) \rightarrow H^{1}\left(X, \ell_{*} M\right) \rightarrow \quad \oplus_{x \in X \backslash U} H_{D R}^{1}\left(\operatorname{Spec} \widehat{K}_{x}, M \otimes \widehat{K}_{x}\right) \rightarrow 0 .
$$

Let $j_{\eta}: \operatorname{Spec}(k(X)) \rightarrow X, j_{\eta, U}: \operatorname{Spec}(k(X)) \rightarrow U$ be the inclusions of the generic point. Then, since $M$ is assumed smooth on $U$,

$$
\left(j_{\eta}\right)_{! *} j_{\eta, U}^{*} M=\ell_{! *} M
$$

One defines (see [12], (3.0.2))

Definition 4.2. Let $M$ be a smooth connection on $U \stackrel{j}{\hookrightarrow} \mathbb{A}^{1}$. Set $\ell=k \circ j$ where $\mathbb{A}^{1} \stackrel{k}{\hookrightarrow} \mathbb{P}^{1}$. Then the index of rigidity of $M$ is defined by $\operatorname{rig}(M)=\chi\left(\mathbb{P}^{1}, \ell_{! *} \mathcal{E} n d(M)\right)$.

Theorem 4.3 (Compare [12], Theorem 3.0.3.). Let $M$ be a holonomic $\mathcal{D}$-module on $\mathbb{A}^{1}$. We assume that $M$ as well as its Fourier transform $\mathcal{F}(M)$ are the middle extensions of their restriction to the generic point. Then one has

$$
\operatorname{rig}(M)=\operatorname{rig}(\mathcal{F}(M)) \text {. }
$$


Proof. Once one has established the equivalences of category in section 3, the proof is exactly the same as Katz's proof in the $\ell$-adic case. Let us just give an outline. We assume $M$ is smooth on $U \stackrel{j}{\hookrightarrow} \mathbb{A}^{1}$ as above, and we write $T=\mathbb{A}^{1} \backslash U$.

Lemma 4.4. We have an exact sequence ( $z_{s}$ is a local coordinate at $s$ )

$$
\begin{aligned}
0 & \rightarrow j_{!_{*}} \mathcal{E} n d(M) \rightarrow j_{! *} M \otimes j_{!_{*}} M^{\vee} \rightarrow \\
& \oplus_{s \in T} i_{s *}\left[\mathcal{E} n d\left(M \otimes k\left(\left(z_{s}\right)\right)\right)^{\nabla} /\left(M \otimes k\left(\left(z_{s}\right)\right)\right)^{\nabla} \otimes\left(M^{\vee} \otimes k\left(\left(z_{s}\right)\right)\right)^{\nabla}\right] \rightarrow 0
\end{aligned}
$$

Proof. The issue is local around the singular points, so we may consider $M$ a connection on $k((t)), j: \operatorname{Spec} k((t)) \hookrightarrow \operatorname{Spec} k[[t]]$. We have by ([13] Proposition 2.9.8 p. 65) $(\delta:=k((t)) / k[[t]]=\mathcal{D} / \mathcal{D} t$, where $\mathcal{D}$ denotes differential operators on $k[[t]]$. The superscript ${ }^{\vee}$ means dual in the appropriate sense.)

$$
0 \rightarrow j_{! *} M \rightarrow j_{*} M \rightarrow\left(\left(M^{\vee}\right)^{\nabla}\right)^{\vee} \otimes \delta \rightarrow 0 .
$$

Now $j_{!_{*}} M$ is $k[[t]]$-torsion-free and hence flat. Also $j_{*} M \otimes j_{!_{*}} M^{\vee}=j_{*} \mathcal{E} n d(M)$. Replacing $M$ with $M^{\vee}$ in (4.4) and tensoring the resulting sequence with $\left(\left(M^{\vee}\right)^{\nabla}\right)^{\vee} \otimes \delta$ yields

$$
\begin{aligned}
\left(\left(M^{\vee}\right)^{\nabla}\right)^{\vee} \otimes \delta \otimes j_{! *} M^{\vee} \cong\left(\left(M^{\vee}\right)^{\nabla}\right)^{\vee} \otimes_{k}\left(M^{\nabla}\right)^{\vee} & \otimes_{k} \operatorname{Tor}_{1}^{\mathcal{O}}(\delta, \delta) \cong \\
& \left(\left(M^{\vee}\right)^{\nabla}\right)^{\vee} \otimes_{k}\left(M^{\nabla}\right)^{\vee} \otimes_{k} \delta
\end{aligned}
$$

We get, therefore, an exact sequence

$$
0 \rightarrow j_{! *} M \otimes j_{! *} M^{\vee} \rightarrow j_{*} \mathcal{E} n d(M) \rightarrow\left(\left(M^{\vee}\right)^{\nabla}\right)^{\vee} \otimes_{k}\left(M^{\nabla}\right)^{\vee} \otimes_{k} \delta \rightarrow 0
$$

Now $j_{!_{*}} \mathcal{E} n d(M)$ is characterized as a sub- $\mathcal{D}$-module of $j_{*} \mathcal{E} n d(M)$ extending $\mathcal{E} n d(M)$ and having $\operatorname{Hom}_{\mathcal{D}}\left(j_{!_{*}} \mathcal{E} n d(M), \delta\right)=(0)$ (cf. op. cit. Lemma 2.9.1, p. 57). This implies by (4.6) that $j_{! *} \mathcal{E} n d(M) \subset j_{! *} M \otimes j ! * M^{\vee}$. We get a diagram

$$
\begin{aligned}
& 0 \longrightarrow \quad j_{!_{*}} \mathcal{E} n d(M) \quad \longrightarrow j_{*} \mathcal{E} n d(M) \longrightarrow \quad \mathcal{E} n d(M)^{\nabla} \otimes \delta \quad \longrightarrow 0 \\
& 0 \longrightarrow j_{! *} M \otimes j_{! *} M^{\vee} \longrightarrow j_{*} \mathcal{E} n d(M) \longrightarrow\left(\left(M^{\vee}\right)^{\nabla}\right)^{\vee} \otimes_{k}\left(M^{\nabla}\right)^{\vee} \otimes_{k} \delta \longrightarrow 0
\end{aligned}
$$

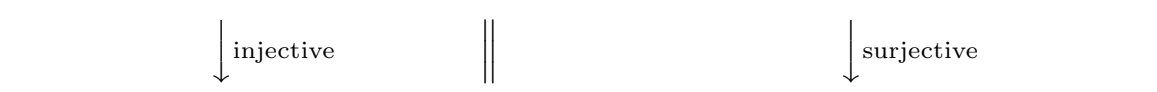

Finally, this yields

$$
\begin{gathered}
0 \rightarrow j_{!_{*}} \mathcal{E} n d(M) \rightarrow j_{!_{*}} M \otimes j_{!_{*}} M^{\vee} \rightarrow V \otimes_{k} \delta \rightarrow 0 \\
V:=\left[\mathcal{E} n d(M)^{\nabla} /\left(M^{\vee}\right)^{\nabla} \otimes_{k} M^{\nabla}\right]^{\vee},
\end{gathered}
$$

proving the lemma.

Returning to the proof of the theorem, we deduce from the lemma

$$
\begin{aligned}
& \operatorname{rig}(M)=h^{0}\left(\mathcal{E} n d\left(M \otimes_{k[t]} k((z))\right)\right)+\chi\left(\mathbb{A}^{1}, j_{!_{*}} \mathcal{E} n d(F)\right)= \\
& \chi\left(\mathbb{A}^{1}, j_{! *} M \otimes j_{! *} M^{\vee}\right)+h^{0}\left(\mathcal{E} n d\left(M \otimes_{k[t]} k((z))\right)\right)+ \\
& \sum_{x \in \mathbb{A}^{1} \backslash U} h^{0}\left(\mathcal{E} n d\left(M \otimes_{k[t]} k((t-x))\right)^{\nabla}\right) /\left[M \otimes_{k[t]} k((t-x))\right]^{\nabla} \otimes\left[M^{\vee} \otimes_{k[t]} k((t-x))\right]^{\nabla} .
\end{aligned}
$$


Here we write $t$ for the coordinate on $\mathbb{A}^{1}$ and $z=\frac{1}{t}$.

Writing $D$ for Verdier dual, so for example $D M=M^{\vee}$, we have that $D$ commutes with middle extension (op. cit. Corollary 2.9.1.2) so

$$
\chi\left(\mathbb{A}^{1}, j_{! *} M \otimes j_{! *} M^{\vee}\right)=\chi\left(\mathbb{A}^{1}, j_{! *} M \otimes D j_{! *} M\right)
$$

Lemma 4.5 (cf. [12], Thm. 3.0.4). Let $N$ be a holonomic $\mathcal{D}$-module on $\mathbb{A}^{1}$. Then

$$
\chi\left(\mathbb{A}^{1}, N \otimes D N\right)=\chi\left(\mathbb{A}^{1}, \mathcal{F}(N) \otimes D \mathcal{F}(N)\right)
$$

Proof. Let $\mathcal{M}$ be a holonomic $\mathcal{D}$-module on $\mathbb{A}^{1}$ and let $k: \mathbb{A}^{1} \hookrightarrow \mathbb{P}^{1}$. Define $\pi: k_{!} \mathcal{M} \rightarrow k_{*} \mathcal{M}$ to be the natural map. By Kashiwara's theorem, ker $\pi$ and coker $\pi$ have the form $\delta^{\oplus n}$. It is not hard to show, e.g. by using the Levelt classification for formal $\mathcal{D}$-modules, that the $n$ is the same for ker and coker, so in particular

$$
\chi\left(\mathbb{A}^{1}, \mathcal{M}\right)=\chi\left(\mathbb{P}^{1}, k_{*} \mathcal{M}\right)=\chi\left(\mathbb{P}^{1}, k_{!} \mathcal{M}\right)=: \chi_{c}\left(\mathbb{A}^{1}, \mathcal{M}\right) .
$$

Let $D_{-}=[x \mapsto-x]^{*} \circ D$. Then $D_{-} \circ \mathcal{F}=\mathcal{F} \circ D$. Also, write $M_{1} *_{!_{+}} M_{2}:=p_{!}\left(M_{1} \otimes M_{2}\right)$, where $p: \mathbb{A}^{1} \times \mathbb{A}^{1} \rightarrow \mathbb{A}^{1}$ is the addition map. One has

$$
\mathcal{F}\left(M_{1} * !+M_{2}\right)=\mathcal{F}\left(M_{1}\right) \otimes \mathcal{F}\left(M_{2}\right)
$$

(cf.[13], 12.2.3(5). To get a formula involving $*_{\text {!* }}$ one must modify the argument given there, replacing the lower star pushforward with lower shriek, and the upper shriek pullback with upper star. By standard theory, the Fourier transform can be computed either with lower star or lower shriek.) Finally using that lower shriek commutes with passage to the fibres, we find

$$
\begin{aligned}
& \chi_{c}\left(\mathbb{A}^{1}, \mathcal{F}(N) \otimes D \mathcal{F}(N)\right)=\operatorname{rank}_{0}\left(\mathcal{F}(N) *_{!+} D_{-} \mathcal{F}(N)\right)= \\
& \operatorname{rank}_{0}\left(\mathcal{F}(N) *_{+} \mathcal{F}(D N)\right)=\operatorname{rank}_{0}(\mathcal{F}(N \otimes D N))=\chi_{c}(N \otimes D N) .
\end{aligned}
$$

For a holonomic $\mathcal{D}$-module $M$ on $\mathbb{A}^{1}$ with coordinate $t=1 / z$, it will be convenient to write $\widetilde{M}:=M \otimes k((z))$. As in Katz, we may write $\widetilde{M}=\widetilde{M} \leq 1 \oplus \widetilde{M}^{>1}$ according to slopes ([15], Thm. 1.5(2), p. 45). One has, since horizontal endomorphisms respect slopes

$$
h^{0}(\mathcal{E} n d(\widetilde{M}))=h^{0}\left(\mathcal{E} n d\left(\widetilde{M}^{\leq 1}\right)\right)+h^{0}\left(\mathcal{E} n d\left(\widetilde{M}^{>1}\right)\right) .
$$

One has by Proposition 3.12 (iv), (v)

$$
h^{0}\left(\mathcal{E} n d\left(\widetilde{M}^{>1}\right)\right) h^{0}\left(\mathcal{E} n d\left(=\widetilde{\mathcal{F} M}^{>1}\right)\right) .
$$

One has, by Definition 4.2 (here we write $M=j_{! *} F$ for $F$ a cnnection on $U$ )

$$
\operatorname{rig}(M)=h^{0}(\mathcal{E} n d(\widetilde{M}))+\chi\left(\mathbb{A}^{1}, j_{! *} \mathcal{E} n d(F)\right) .
$$


This yields

$$
\begin{aligned}
& \operatorname{rig}(M)=\chi\left(\mathbb{A}^{1}, j_{! *} F \otimes j_{! *} F^{\vee}\right)+h^{0}(\mathcal{E} n d(\widetilde{M}))+ \\
& \quad+\sum_{x \in \mathbb{A}^{1}} \operatorname{dim}\left[\mathcal{E} n d_{\nabla}(\widetilde{M}) / \widetilde{M}^{\nabla} \otimes\left(\widetilde{M}^{\vee}\right)^{\nabla}\right] .
\end{aligned}
$$

Since $D$ commutes with middle extensions, we have

$$
\chi\left(\mathbb{A}^{1}, M \otimes D(M)\right)=\chi\left(\mathbb{A}^{1}, j_{! *} F \otimes j_{! *} F^{\vee}\right)
$$

where $D(M)$ is the Verdier dual of $M$. By lemma 4.5

$$
\chi\left(\mathbb{A}^{1}, M \otimes D(M)\right)=\chi\left(\mathbb{A}^{1}, \mathcal{F}(M) \otimes D \mathcal{F}(M)\right) .
$$

We claim the following identities (4.21) - (4.23)

$$
\begin{gathered}
h^{0} \mathcal{E} n d\left(\widetilde{M}^{>1}\right)=h^{0} \mathcal{E} n d\left(\widetilde{\mathcal{F}(M)}^{>1}\right) \\
h^{0} \mathcal{E} n d(\widetilde{M} \leq 1)= \\
\sum_{x \in \mathbb{A}^{1}} \operatorname{dim}\left[\mathcal{E}=n d_{\nabla}(\widetilde{\mathcal{F}(M)}) / \widetilde{\mathcal{F}(M)}^{\nabla} \otimes\left(\widetilde{\mathcal{F}(M)}^{\vee}\right)^{\nabla}\right] \\
\sum_{x \in \mathbb{A}^{1}} \operatorname{dim}\left[\mathcal{E} n d_{\nabla}(\widetilde{\mathcal{M}}) / \widetilde{M}^{\nabla} \otimes\left(\widetilde{M}^{\vee}\right)^{\nabla}\right] .
\end{gathered}
$$

(Here ${ }^{\leq 1}$ and ${ }^{>1}$ refer to the slope decomposition.) Now (4.21) follows immediately from proposition $3.12 \mathrm{iv}$ ), v), and (4.22) is equivalent to (4.23). Finally, (4.23) follows from Proposition 3.10, from Remark 2.6 which implies that for $x \neq x^{\prime} \in \mathbb{A}^{1}$,

$$
\operatorname{Hom}\left(\mathcal{F}\left(M \otimes_{k[t]} k\left(\left(t_{x}\right)\right)\right), \mathcal{F}\left(M \otimes_{k[t]} k\left(\left(t_{x^{\prime}}\right)\right)\right)\right)=0,
$$

and from the following lemma.

Lemma 4.6 (Compare [12], Proposition 3.1.8). Let $M$ be a connection on $k((t))$. Then one has

$$
\mathcal{E} n d_{\nabla}(M) / M^{\nabla} \otimes\left(M^{\vee}\right)^{\nabla}=\mathcal{E} n d_{\nabla}\left(M / M^{\nabla} \otimes_{k} \mathcal{O}\right) .
$$

Proof. We consider the isotypical decomposition $\oplus_{N} M_{N}$ of $M$, with $\operatorname{Hom}\left(N, N^{\prime}\right)=0$ if $N \neq N^{\prime}$. Let us write it as $M^{\prime} \oplus M_{\mathcal{O}}$ with $M^{\prime}=\oplus M_{N}$ where this sum is over $N \neq \mathcal{O}$. Then $h^{0}\left(M^{\prime}\right)=0, \operatorname{Hom}\left(M^{\prime}, \mathcal{M}_{\mathcal{O}}\right)=0$ thus the left hand side (LHS) of (4.24) fulfills

$$
\operatorname{LHS}(M)=\operatorname{LHS}\left(M^{\prime}\right)+\operatorname{LHS}\left(M_{\mathcal{O}}\right) .
$$


And the same holds true for the right hand side (RHS)

$$
R H S(M)=R H S\left(M^{\prime}\right)+R H S\left(M_{\mathcal{O}}\right) .
$$

Moreover, $L H S\left(M^{\prime}\right)=R H S\left(M^{\prime}\right)$ as $M^{\prime}$ has no flat sections. Thus we reduce the computation to $M=M_{\mathcal{O}}$, that is $M$ is nilpotent. In this case, this is a purely linear algebra problem. We write $M=\oplus_{i} M_{i}$ where $M_{i}$ is a maximal Jordan block. Then $M_{i}^{\nabla}=k$ and we set $N_{i}=M_{i} / \mathcal{O}$. Then (4.24) is equivalent to saying that for $i, j$, one has an exact sequence

$$
0 \rightarrow \mathcal{O} \rightarrow \operatorname{Hom}\left(M_{i}, M_{j}\right) \rightarrow \operatorname{Hom}\left(N_{i}, N_{j}\right) \rightarrow 0 .
$$

We recall, in the context of $\mathcal{D}$-modules, the central beautiful observation of Katz.

TheOREM 4.7. Let $X$ be a smooth, complete curve. Let $U \stackrel{j}{\hookrightarrow} X$ be Zariski open, and let $M$ be an irreducible connection on $U$. Suppose

$$
\chi\left(X, j_{! *} \mathcal{E} n d(M)\right) \geq 2 .
$$

Let $M^{\prime}$ be another irreducible connection on $U$, and assume for all $x \in X \backslash U$ we have $M \otimes \widehat{K}_{x} \cong M^{\prime} \otimes \widehat{K}_{x}$, where $\widehat{K}_{x}$ is the Laurent series field at $x$. Then $M \cong M^{\prime}$.

Proof. The point is that $\chi\left(X, j_{!_{*}} N\right)$ for $N$ a connection on $U$ depends only on $X$ and the $N \otimes \widehat{K}_{x}$. (See e.g. [15], thm. 4.9, p. 69.) In particular,

$$
\begin{aligned}
2 \leq \chi\left(X, j_{! *} \mathcal{E} n d(M)\right) & =\chi\left(X, j_{! *} \mathcal{H o m}\left(M, M^{\prime}\right)\right) \\
& \leq h^{0}\left(X, j_{! *} \mathcal{H o m}\left(M, M^{\prime}\right)\right)+h^{2}\left(X, j_{! *} \mathcal{H o m}\left(M, M^{\prime}\right)\right) .
\end{aligned}
$$

Since Verdier duality for holonomic $\mathcal{D}$-modules on a complete smooth variety commutes with the de Rham functor (cf. [4] (5), p. 326), we obtain

$$
h^{2}\left(X, j_{!_{*}} \mathcal{H o m}\left(M, M^{\prime}\right)\right)=h^{0}\left(X, j_{! *} \mathcal{H o m}\left(M^{\prime}, M\right)\right) .
$$

It follows that at least one of the modules $\mathcal{H o m}\left(M, M^{\prime}\right), \mathcal{H o m}\left(M^{\prime}, M\right)$ has a nontrivial horizontal section. By irreducibility, the two modules are necessarily isomorphic.

Recall Katz' definition ([12], Introduction).

Definition 4.8. Let $j: U \hookrightarrow X$ be as above, and let $M$ be an irreducible connection on $U$. We say that $M$ is rigid if $M^{\prime}$ an irreducible connection on $U$ and $M^{\prime} \otimes \widehat{K}_{x} \cong M \otimes \widehat{K}_{x}$ for all $x \in X \backslash U$ implies $M \cong M^{\prime}$.

Corollary 4.9 (of Theorem 4.3). Let $M$ be a rank 1 meromorphic connection on $\mathbb{P}^{1}$ and assume the slope of $M$ at $\infty$ is $>1$. Then $\mathcal{F}(M)$ is rigid.

Proof. $\mathcal{F}(M)$ is smooth on $\mathbb{A}^{1}([15],(1.4)(\mathrm{b})$, p. 78). Since $\operatorname{End}(M)=\mathcal{O}$, the hypotheses of Theorem 4.3 are satisfied, and we conclude

$$
\operatorname{rig}(\mathcal{F}(\mathrm{M}))=2 .
$$

The result now follows from Theorem 4.7. 
THEOREM 4.10. Let $X$ be a smooth, complete curve, and let $j: U \hookrightarrow X$ be a non-empty open affine. Let $M$ be an irreducible, rigid connection on $U$. Then $\operatorname{rig}(\mathrm{M})=2$.

Proof. By irreducibility and duality,

$$
h^{0}\left(j_{!_{*}} \mathcal{E} n d(M)\right)=h^{2}\left(j_{! *} \mathcal{E} n d(M)\right)=1,
$$

so the assertion is equivalent to $h^{1}\left(j_{!_{*}} \mathcal{E} n d(M)\right)=0$. Let $\mathcal{C}$ be the category of augmented, artinian, local $k$-algebras. Consider the functor $F: \mathcal{C} \rightarrow$ sets,

$$
\begin{aligned}
F(R)=\left\{\left(\mathcal{M}, \nabla_{\mathcal{M} / R}\right) \operatorname{lifting}(M, \nabla) \mid\right. & \\
& \left.\left(\widehat{\mathcal{M}}, \nabla_{\widehat{\mathcal{M}} / R}\right) \cong\left(\widehat{M}, \nabla_{\widehat{M}}\right) \otimes_{k} R\right\} / \text { isom. }
\end{aligned}
$$

Here the $\widehat{ }$ means restriction to $\widehat{U}$, the product of power series fields at points of $X \backslash U$. We will show that this functor is effectively pro-representable and smooth, with tangent space $H^{1}\left(j_{!_{*}} \mathcal{E} n d(M)\right)$. Using a criterion of Artin, [1], we will show there exists a pointed affine scheme $(S=\operatorname{Spec} A, 0)$, smooth and of finite type over $k$, and a connection $\left(N, \nabla_{N}\right)$ on $U \times S$ relative to $S$ such that

1. $\left.\left(N, \nabla_{N}\right)\right|_{0} \cong\left(M, \nabla_{M}\right)$.

2. $\left(\widehat{N}, \nabla_{\widehat{N}}\right) \cong\left(\widehat{M}, \nabla_{\widehat{M}}\right) \times S$, where $\widehat{N}$ denotes the restriction of $N$ to the union of tubes $\operatorname{Spec} A\left(\left(t_{x}\right)\right)$, where $x \in X \backslash U$ and $t_{x}$ is a local parameter at $x$.

3. $\widehat{\mathcal{O}}_{S, 0}$ pro-represents the functor $F$, and $\left(N, \nabla_{N}\right)$ is universal.

Assuming for a moment that we have $\left(N, \nabla_{N}\right)$ satisfying these conditions, consider the connection $H:=\mathcal{H o m}\left(M \otimes_{k} \mathcal{O}_{S}, N\right)$. By rigidity, for any point $s \in S$, the connection on $H \otimes \mathcal{O}_{S} k(s)$ has a horizontal section. In particular, this is the case at the generic point, so there will exist an nonempty open $T \subset S$ and a horizontal isomorphism $\left.M \otimes_{k} \mathcal{O}_{T} \cong N\right|_{T}$. To prove the theorem, we need to show that $N \otimes_{\mathcal{O}_{S}}$ $k[\epsilon] \cong M \otimes_{k} k[\epsilon]$ for any $\tau:$ Spec $k[\epsilon] \hookrightarrow S$ centered at 0 . If $0 \in T$ this is clear. If not, we choose a smooth curve $C \hookrightarrow=S$ passing through 0 and tangent to $\tau$. We can further assume $C \cap T \neq \emptyset$. Shrinking $S$ to a neighborhood of 0 , we can assume $0 \in C$ is defined by $f=0$ and $C \cap T=C \backslash\{0\}$. Restricting the above horizontal isomorphism to $C \backslash\{0\}$ and multiplying by a power of $f$, we get a horizontal injection $i:\left.M \otimes_{k} \mathcal{O}_{C} \hookrightarrow N\right|_{C}$. Since $\left.N\right|_{C}$ is a coherent sheaf on $U \times C$ we see that $\left.\cap f^{n} N\right|_{C}=(0)$. Scaling $i$ by an appropriate power of $f$ we can therefore suppose that the restriction to $0, i_{0}:=M \rightarrow N_{0} \cong M$ is not zero. But $M$ is assumed irreducible, so this map is necessarily an isomorphism. It follows that $\left.M \otimes_{k} \mathcal{O}_{C} \cong N\right|_{C}$, so in particular, $\tau^{*} N \cong M \otimes_{k} k[\epsilon]$ as desired.

It remains to show the existence of $S$. Consider a diagram in $\mathcal{C}$

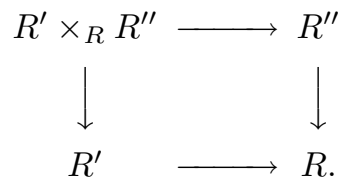

Note that, by irreducibility, any flat automorphism of a lifting $\mathcal{M}$ of $M$ over $R$ is necessarily constant (i.e. in $R^{\times}$.) Suppose given $\mathcal{M}^{\prime} \in F\left(R^{\prime}\right)$ and $\mathcal{M}^{\prime \prime} \in F\left(R^{\prime \prime}\right)$ which agree in $F(R)$, i.e. there is a flat isomorphism $\mathcal{M}^{\prime} \otimes R \cong \mathcal{M}^{\prime \prime} \otimes R$. Fix such an isomorphism $\phi$. By the above, it is unique upto $R^{\times}$. Consider a subsheaf $\mathcal{N} \subset \mathcal{M}^{\prime} \times \mathcal{M}^{\prime \prime}, \mathcal{N}=\left\{\left(m^{\prime}, m^{\prime \prime}\right) \mid \phi\left(m^{\prime} \otimes R\right)=m^{\prime \prime} \otimes R\right\}$. Clearly, $\mathcal{N}$ is a relative 
connection on $U \times \operatorname{Spec}\left(R^{\prime} \times_{R} R^{\prime \prime}\right)$ lifting $\mathcal{M}^{\prime}$ and $\mathcal{M}^{\prime \prime}$. Assuming one of the rings $R^{\prime}$ and $R^{\prime \prime}$ surjects onto $R$, elements in $R^{\times}$lift to say $R^{\prime}$. We can then modify $\phi$ by an automorphism of $\mathcal{M}^{\prime}$. In this way we see that $\mathcal{N} \in F\left(R^{\prime} \times_{R}=R^{\prime \prime}\right)$ is independent of the choice of $\phi$. Schlessinger's criterion [18]

$$
F\left(R^{\prime} \times_{R} R^{\prime \prime}\right) \cong F\left(R^{\prime}\right) \times_{F(R)} F\left(R^{\prime \prime}\right)
$$

is therefore satisfied. The tangent space is easily computed to be

$$
\operatorname{ker}\left(H_{D R}^{1}(U, \mathcal{E} n d(M)) \rightarrow H_{D R}^{1}(\widehat{U}, \mathcal{E} n d(M)) \cong H^{1}\left(X, j_{! *} \mathcal{E} n d(M)\right),\right.
$$

(Compare Remark 4.1.) and it follows again by [18] that $F$ is prorepresentable.

Similarly, the obstruction to smoothness lies in $H^{2}\left(X, j_{!_{*}} \mathcal{E} n d(M)\right)$. Again by irreducibility, the trace map

$$
H^{2}\left(X, j_{! *} \mathcal{E} n d(M)\right) \rightarrow H^{2}\left(X, j_{! *} \mathcal{O}_{U}\right) \cong k
$$

is an isomorphism. Clearly, this trace carries the obstruction to lifting the connection to the corresponding obstruction to lifting the determinant of the connection. But these determinant connections are parametrized by a smooth groupscheme so the determinant obstruction vanishes. We conclude that our deformation functor $F$ is smooth.

To construct our family $\mathcal{M}_{S}$ of connections algebraizing the above formal moduli, we apply Artin's criterion [1], Thm. 1.6. For this, we need to show our functor $F$ is effective and of finite presentation. Effectivity means that if $F$ is pro-represented by $\Lambda$, then there exists $\mathcal{M}_{\Lambda} \in F(\Lambda)$ restricting to the representing object in $\lim _{\longleftarrow} F\left(\Lambda / \mathfrak{m}_{\Lambda}^{n}\right)$. Choose a lattice $\mathcal{L} \subset \widehat{M}$ which is stable under the group of horizontal automorphisms of $\widehat{M}$. (Let $\mathcal{L}_{0} \subset \widehat{M}$ be any lattice. Let $e_{1}, \ldots, e_{n}$ be a vector space basis for the ring of horizontal endomorphisms of $\widehat{M}$. Then $\mathcal{L}:=\sum e_{i} \mathcal{L}_{0}$ works.) Let $\mathcal{M}_{n} \in F\left(\Lambda / \mathfrak{m}^{n}\right)$ be

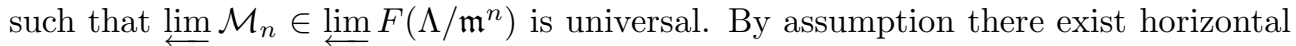
isomorphisms $\psi_{n}: \overleftrightarrow{M \otimes} \Lambda / \mathfrak{m}^{n} \cong \widehat{\mathcal{M}}_{n}$ at infinity. We may glue $\mathcal{M}_{n}$ on $U \times \operatorname{Spec}\left(\Lambda / \mathfrak{m}^{n}\right)$ to $\psi_{n}\left(\mathcal{L} \otimes_{k} \Lambda / \mathfrak{m}^{n}\right)$ to get bundles $\overline{\mathcal{M}}_{n}$ on $X \times \operatorname{Spec}\left(\Lambda / \mathfrak{m}^{n}\right)$. Since $\operatorname{End}_{\nabla}\left(\widehat{M} \otimes \Lambda / \mathfrak{m}^{n}\right)=$ $\operatorname{End}_{\nabla}(\widehat{M}) \otimes \Lambda / \mathfrak{m}^{n}$, the automorphism $\psi_{n}^{-1} \circ\left(\psi_{n+1} \otimes \Lambda / \mathfrak{m}^{n}\right)$ stabilizes $\mathcal{L} \otimes \Lambda / \mathfrak{m}^{n}$. It follows that the $\overline{\mathcal{M}}_{n}$ are compatible. By Grothendieck, there exists $\overline{\mathcal{M}}$ on $X \times \operatorname{Spec} \Lambda$ which induces the $\overline{\mathcal{M}}_{n}$. The connections on the $\overline{\mathcal{M}}_{n}$ correspond to splittings of the Atiyah sequences, with bounded poles on $X \backslash U$ corresponding to the fact that the connection on $\widehat{M}$ does not stabilize $\mathcal{L}$. Again, these splittings agree, so we get a connection on $\overline{\mathcal{M}}$. To examine the polar behavior of this connection, let $m \geq 0$ be such that $\nabla(\mathcal{L}) \subset \mathcal{L}(m(X \backslash U)) \otimes \Omega_{X}^{1}$. Then the connection on $\overline{\mathcal{M}}$ has poles of order $\leq m$ on $(X \backslash U) \times \operatorname{Spec} \Lambda$. Both effectivity and finite presentation follow from this.

The existence of $S, \mathcal{M}_{S}$ satisfying properties 1-3 above follows from Artin, proving the theorem. $\square$

REMARKS 4.11. Theorems 4.7 and 4.10 together give a cohomological criterion for rigidity of connections. This criterion is proven by Katz in [12], section 1, for regular singular connections, using transcendental methods. It is unknown on the $\ell-$ adic side. Note also one does not assume $X$ to be $\mathbb{P}^{1}$ in the proofs, yet we know ([12], section 1) that rigidity is meaningful only on $\mathbb{P}^{1}$. Indeed, over a curve $X$ of genus $>0$, we can deform $M$ by twisting with a family of global rank 1 connections. The sheaf $j_{! *} \mathcal{E} n d(M)$ contains $\mathcal{O}_{X}$ as a direct summand, so $\operatorname{rig}(M)=2$ implies $X=\mathbb{P}^{1}$. 


\section{REFERENCES}

[1] Artin, M., Algebrization of Formal Moduli I, in Global Analysis, edited by D.C.Spencer and S. Iyanaga, Princeton University Press (1969), pp. 21-71.

[2] Beilinson, A.; Bloch, S.; Esnault, H., $\epsilon$-factors for Gauß-Manin determinants, Moscow Mathematical Journal, 2:3 (2002), pp. 477-532.

[3] Beilinson, A.; Bloch, S.; Deligne, P.; Esnault, H., work in progess.

[4] Borel, A. ET Al., Algebraic D-Modules, Perspectives in Math. Vol. 2, Academic Press (1987).

[5] Deligne, P., Équations Différentielles à Points Singuliers Réguliers, Lectures Notes in Mathematics 163, Springer Verlag.

[6] Deligne, P., Le formalisme des cycles évanescents, Exposé XIII, Exposé XIV, SGA 7 II, Lectures Notes in Mathematics 340, pp. 82-115.

[7] Garcia Lopez, R., Microlocalization and stationary phase, 32 pages, this volume.

[8] Grothendieck, A., Étude cohomologique des faisceaux cohérents, III, in Élements de Géométrie Algébrique, Publ. Math. IHES 11 (1961).

[9] Katz, N., Local-to-global extensions of representations of fundamental groups, Ann. Inst. Fourier, Grenoble 36:4 (1986), pp. 69-106.

[10] Katz, N., On the calculation of some differential Galois groups, Inv. Math., 87 (1986), pp. 13-61.

[11] Katz, N.; Laumon, G., Tranformations de Fourier et majoration de sommes exponentielles, Publ. Math. IHES, 62 (1985), pp. 361-202.

[12] Katz, N., Rigid Local Systems, Annals of Mathematical Studies, 139, Pinceton University Press (1996).

[13] Katz, N., Exponential Sums and Differential Equations, Annals of Mathematical Studies 124, Pinceton University Press (1996).

[14] Laumon, G., Transformation de Fourier, constantes d'équations fonctionnelles et conjecture de Weil, Publ. Math. IHES, 65 (1987), pp. 131-210.

[15] Malgrange, B., Équations Différentielles à Coefficients Polynomiaux, Progress in Mathematics 96, Birkhäuser (1991).

[16] Malgrange, B., Sur les points singuliers des équations différentielles, Ens. Math., 20 (1974), pp. $149-176$.

[17] Malgrange, B., Modules différentiels et classes de Gevrey, in Mathematical Analysis and Applications, Part B, Adv. in Math. Suppl. Stud., 7b (1981), pp. 513-530.

[18] Schlessinger, M., Functors of Artin Rings, Trans. Amer. Math. Soc. 130 (1968), pp. 205-222.

[19] Zariski, O.; Samuel, P., Commutative Algebra, Vol. II, Van Nostrand (1960). 
